\title{
Graph Based Image Saliency Detection
}

\author{
Ye Huang \\ Institute of Optics and Electronics Chinese Academy of \\ Sciences, Sichuan Province, 610209, China \\ University of Chinese Academy of Sciences, Beijing, \\ 100039, China
}

\begin{abstract}
In this paper, we propose a new saliency detection method based on graph. The method firstly uses SLIC to segment the image into a set of superpixels which are not overlapped, and regards these superpixels as nodes of the graph, then constructs a partly connected graph with these nodes. Secondly, the saliency values of these nodes are computed via absorbing Markov chain and manifold ranking, and a saliency map are obtained corresponding to each image. Finally, it uses a contrast stretching function to correct the above saliency map for improving the quality of image. Experimental results demonstrate that the proposed method can more easily distinguish salient objects from background, and performs better than some methods in terms of robustness and performance.
\end{abstract}

Keywords-Image Saliency; Absorbing Markov chain; Manifold Ranking; Saliency map

\section{INTRODUCTION}

Visual saliency detection can identify the most important region in a complex scene, which attracts human's huge attention rapidly. The method can reduce the complexity of image analysis and improve the accuracy. Visual saliency detection is widely applied to computer vision including image compression, image segmentation, object recognition, image retrieval and so on. Now many researchers are devoted to studying this filed and propose lots of typical algorithms. These in general are categorized as either bottom-up or top-down models. Bottom-up models are data-driven, while top-down model are task-driven.

Existing methods mostly simulated the bottom-up process of biological vision. Itti et al.[1] firstly proposed visual saliency model, which extracted multiscale low-level features including intensity, color and orientation and use center-surround operation to calculate image saliency. Bruce et al.[2] computed saliency based on information maximization. Harel et al.[3] proposed a graph-based solution to obtain a saliency map. Zhang et al.[4] proposed a bayesian framework to calculate saliency using natural statistics. Hou et al.[5] firstly detected image saliency in frequency domain by calculating spectrum residual. Achanta et al.[6] computed the saliency of each pixel by considering its color contrast to the entire image. Cheng et al.[7] used global contrast with respect to the entire image and spatial relationships among the regions to compute image saliency. Goferman et al.[8] gave a different definition about saliency and proposed a context-aware saliency method to detect salient object region. Gopalakrishnan et al.[9] exploited the

\author{
Jianlin Zhang, Yuxing Wei \\ Institute of Optics and Electronics Chinese Academy of \\ Sciences, Sichuan Province, 610209, China
}

hitting time on a fully connected and a sparsely connected graph to detect salient region. Recently, Yang et al.[10] transformed saliency detection into a graph-based ranking problem and received a full resolution saliency map.

In this work, we propose a new visual saliency detection method based on graph. Different from existing graph-based methods which usually exploit the equilibrium distribution in an ergodic Markov chain to calculate the saliency, we adopt the properties of absorbing Markov chain, which is similar with [11]. Those methods using the properties of ergodic Markov chain indeed achieved much success in saliency detection. They in general highlighted small regions and boundaries of object well, but cannot highlight entire object uniformly. To solve this problem, we first exploit the properties of absorbing Markov chain. Given an image as a graph, we define Markov chain over the graph and select some absorbing nodes, then treat the expected time that one transient node spend to arrive at an absorbing nodes. However, the results of saliency detection are not always efficient. When the background occupies lots of area of the entire image and some are near the image center, this method cannot suppress background region efficiently. Therefore, we combine manifold ranking [10] and nonlinear correction to make saliency map much better, in which background regions are suppressed and salient objects are highlighted uniformly.

\section{Proposed SAliency Detection Model}

Proposed saliency detection model combines absorbing Markov chain and manifold ranking to measure saliency, including the following steps: firstly, an input image is divided into $k$ superpixels by SLIC algorithm[12] and constructed into a partly connected graph. Secondly, we define an absorbing Markov chain over the image graph to obtain the first saliency map. Thirdly, we select some foreground superpixels as queries and use manifold ranking method to obtain the second saliency map. Finally, we use contrast stretching function to correct above saliency map. The overview of our algorithm is shown in Fig.1.

\section{A. Graph Construction}

We construct an image graph $G$ with superpixels generated by SLIC as nodes $V$ and links between pairs of nodes as edges $E$. Because we adopt the properties of absorbing Markov chain to compute saliency of superpixels, we need to select some virtual background absorbing nodes. Considering that salient regions hardly occupy image 
boundaries, we choose some boundary superpixels as above absorbing nodes. In the partly connected image graph, each node is connected to its adjacent transient nodes and other nodes which share common boundaries with its adjacent nodes. And boundary transient nodes are fully connected with each other. The weight $w_{i j}$ between nodes $i$ and $j$ is defined as

$$
w_{i j}=e^{-\frac{\left\|x_{i}-x_{j}\right\|}{\sigma^{2}}}
$$

Where $x_{i}$ and $x_{j}$ are separately the mean of nodes $i$ and $j$ in the CIE LAB color space, $\sigma$ is a constant that control the strength of the weight, here, we set square of it as 0.1 .

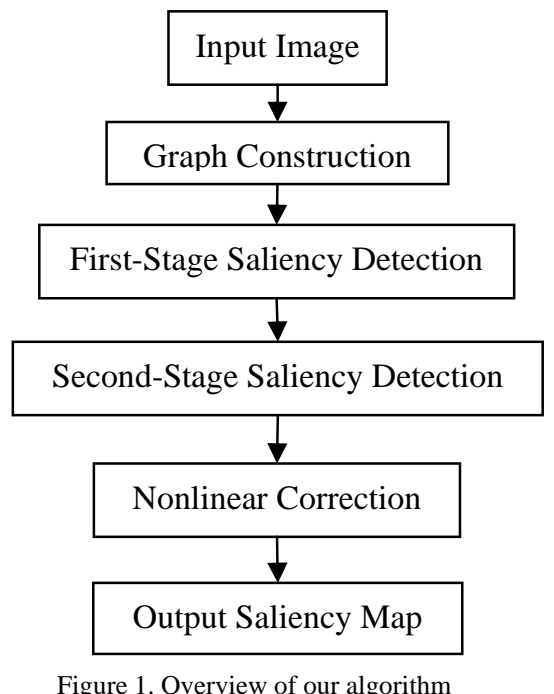

\section{B. First-Stage Saliency detection}

In a Markov chain, there are many states. A state $S_{i}$ is absorbing when the probability of moving from it to any state $S_{j}$ expect itself is zero but to itself is one. If a Markov chain has at least one absorbing state, it is an absorbing Markov chain. Here, we exploit the expected number of times that a transient state spends before being absorbed to compute saliency values. In above image graph, nodes equal states. We first renumber the nodes to make $k$ transient nodes come first and $l$ absorbing nodes follow. Then we define an affinity matrix $A$ used for representing the relationship between nodes as

$$
a_{i j}=\left\{\begin{array}{cc}
w_{i j} & j \in N(i), 1 \leq i \leq k \\
1 & \text { if } i=j \\
0 & \text { otherwise }
\end{array}\right.
$$

Where $N(i)$ denotes the nodes connected to node $i$. Then we define a degree matrix $D$ as

$$
D=\operatorname{diag}\left(\sum_{j} a_{i j}\right)
$$

Thus we can obtain the transition matrix $P$

$$
P=D^{-1} A=\left(\begin{array}{cc}
K & L \\
0 & I
\end{array}\right)
$$

Where $K \in[0,1]^{k \times k}$ contains the transition probabilities between any pair of transient nodes, $L=[0,1]^{k \times l}$ contains the transition probabilities between any transient node to any absorbing node. 0 is the $k \times l$ zero matrix and $I$ is the $l \times l$ identity matrix.

Now transition matrix $P$ is standard which contains a small number of nonzero elements. We can calculate fundamental matrix $N$ of the absorbing chain, $N=(I-K)^{-1}$ where $n_{i j}$ represented the expected number of times that a transient node $i$ needs to transfer into an absorbing node $j$. Supposed $c$ is a $k$ dimensional column vector in which every element is one, we can calculate the absorbed time for each transient node, that is,

$$
y=N \times C
$$

Here, the values of $y$ is first-stage saliency values for superpixels. Here we set the number of superpixels as 250 .

\section{Second-Stage Saliency detection}

The first-stage saliency detection will not suppress the background efficiently when background regions are long-range homogeneous and some occupy the center of image as shown in Fig.2. In order to solve this problem, we adopt manifold ranking method to further detect saliency.

Manifold ranking function $f$ casts each node $S_{i}$ into a ranking value $f_{i}$, viz., $V \rightarrow R^{\mathrm{n}}$. And $f$ can be regarded as a ranking vector $f=\left[f_{1}, f_{2}, \ldots, f_{k}\right]^{T}$. $f=\left[f_{1}, f_{2}, \ldots, f_{k}\right]^{T}$ denotes an indication vector in which $y_{i}=1$, if $i$ is a query, otherwise, $y_{i}=0$.In an image graph, given an affinity matrix $A=\left\{a_{i j}\right\}_{k \times k}$, and a degree matrix $D=\operatorname{diag}\left\{d_{11}, \ldots, d_{k k}\right\}$ where $d_{i i}=\sum_{j} a_{i j}$, The optimal solution of ranking function can be transformed into an optimization problem as follows: 


$$
f^{*}=\underset{f}{\operatorname{argmin}} \frac{1}{2}\left(\sum_{i, j=1}^{k} a_{i j}\left\|\frac{f_{i}}{\sqrt{d_{i i}}}-\frac{f_{j}}{\sqrt{d_{j j}}}\right\|^{2}+\mu \sum_{i=1}^{k}\left\|f_{i}-y_{i}\right\|^{2}\right)(6)
$$

Where $\mu$ controls the balance of smoothness constraint and fitting constraint. By further simplifying above equation, we can obtain the optimal solution as follows

$$
f^{*}=(D-\alpha A)^{-1} y
$$

In this step, we binarize first-stage saliency map by using an adaptive threshold, and select foreground of saliency map as queries. We compute the similarity between other nodes and those queries sequentially. The process is completed by using manifold ranking method. And the optimal solutions are second-stage saliency values.
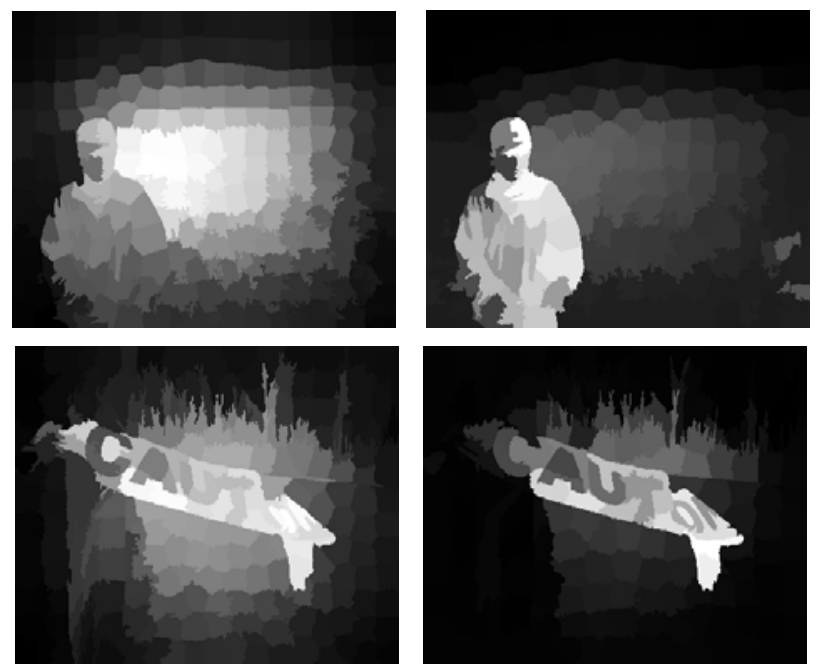

Figure 2. Left: the first-stage saliency map. Right: the second-stage saliency map.

\section{Nonlinear Correction}

The main goal of nonlinear correction is to clarify the junction between salient object and background and keep salient object integrated. Here we adopt a continuous and nonlinear contrast stretching function $S$ defined as

$$
s=\frac{1}{1+(m / r)^{T}}
$$

Where $r \in[0,1], s \in[0,1]$ and $T$ control the function slope, here, we set it as 200. The curve form is shown in Fig.3. The function can suppress regions with lower saliency values and highlight regions with higher values. So the contrast and quality of saliency map are enhanced.

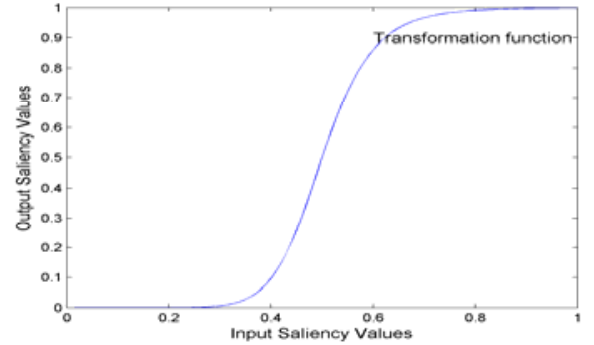

Figure 3. Adopted contrast stretching function curve

\section{EXPERIMENTAL RESULTS}

We evaluate the proposed method on a public dataset [6] which contains 1,000 images with accurate human-labelled ground truth. We compare our method with eight existing methods: the IT[1], LR[13], LC[14], MSS[15], SR[5], FT[6], HC[7], RC[7] methods. Some visual comparison examples are shown as in Fig.5, which show that the proposed method can highlighted salient region uniformly and accurately and suppress the background efficiently.

According to two experimental methods proposed by [6], we can evaluate methods by precision, recall and F-measure. In Fig.4, the left comparison results are obtained by binarizing the saliency map using an adaptive threshold. The right precision-recall curves are obtained by binarizing the saliency map using threshold in the range of 0 and 255. This figure tells that comparing with other methods, our method can obtain much higher precision rate and recall rate. And higher F-measure means that our method has better overall performance in detecting salient regions.
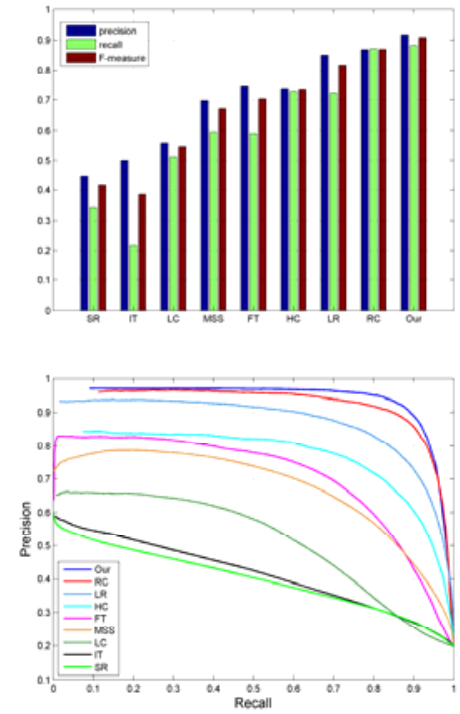

Figure 4. Left: precision, recall and F-measures bars for adaptive threshold. Right: precision-recall curves for all methods. Our method outperforms other methods. 

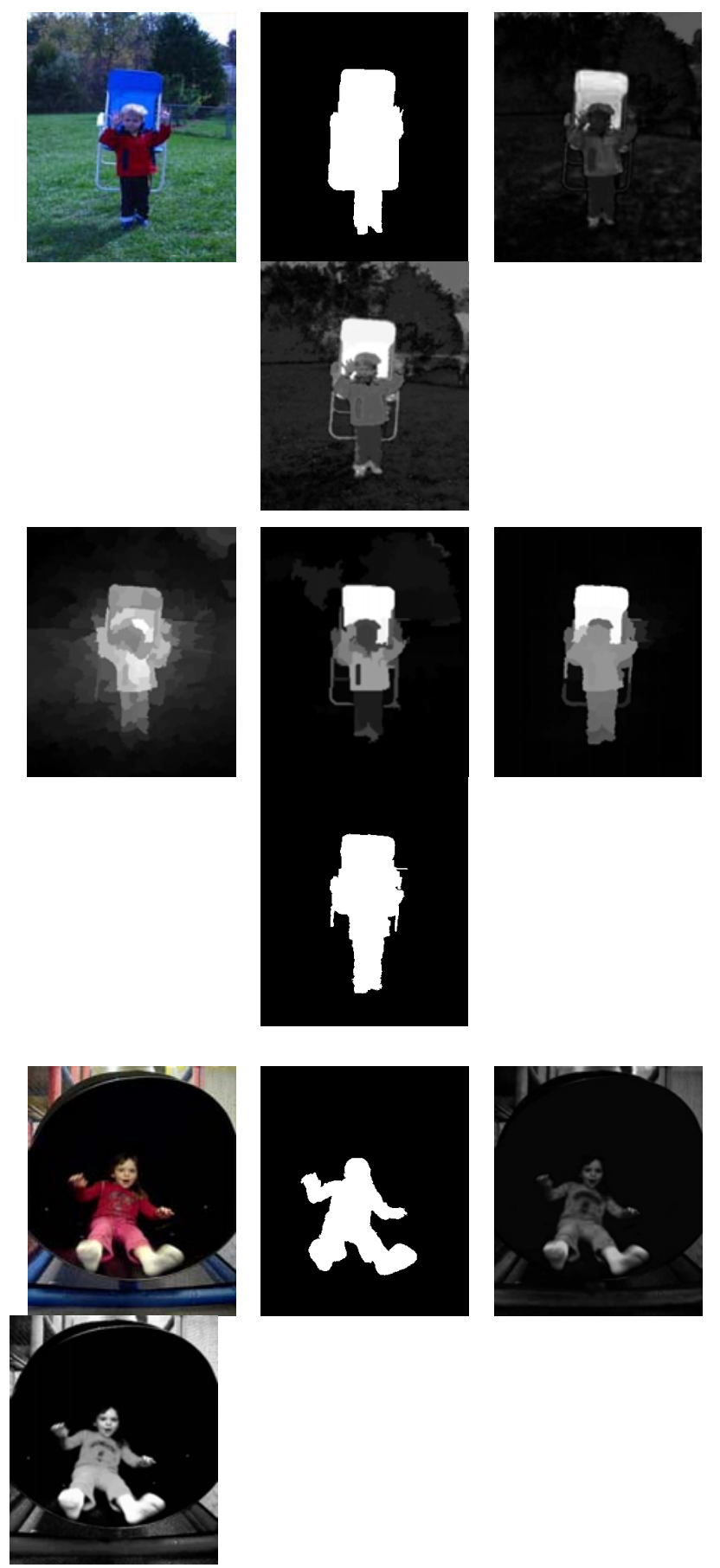
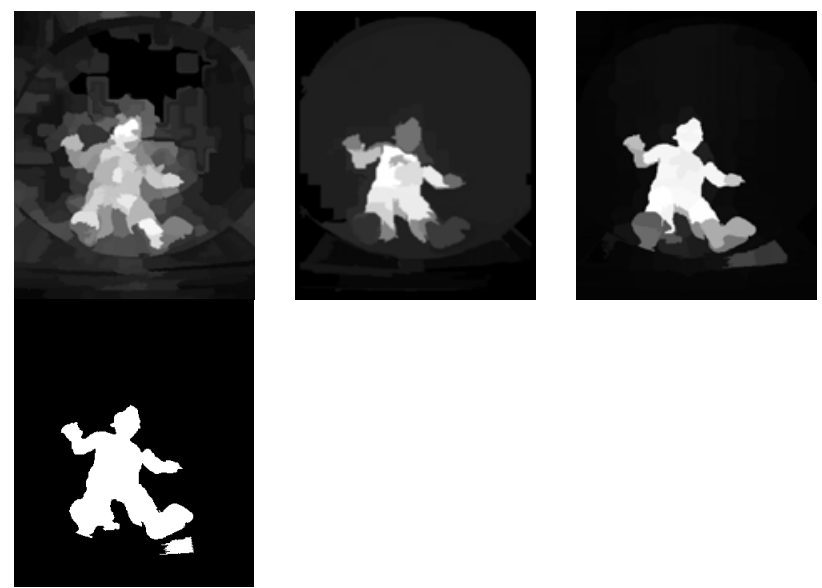

Figure 5. Saliency detection results of different method. From the first image to the last image: Original image, Ground truth, FT, HC, LR, RC, OURS, OURS-Correction. Our saliency maps close to the ground truth.

\section{CONCLUSION}

This paper proposes a new bottom-up image saliency detection method based on graph. The method exploits SLIC to segment an image into some superpixels and regard them as nodes, then constructs a partly-connected graph. The properties of an absorbing Markov chain are applied to compute first-stage saliency map. Next it makes use of manifold ranking function to obtain second-stage saliency map. And it improve the quality of saliency map with nonlinear correction method. The proposed algorithm can suppress background well and highlight salient region uniformly. In the future work, we plan to study other features such as multiscale feature, to further improve the performance of saliency detection, and apply it to dynamic images.

\section{V REFERENCES}

[1] L. Itti, C. Koch, and E. Niebur. A model of saliency-based visual attention for rapid scene analysis. IEEE Trans. on pattern analysis and machine intelligence, vol.20. no.11,1998.

[2] N.Bruce and J. Tsotsos. Saliency based on information maximization. NIPS, 2006.

[3] J. Harel, C. Koch, and P. Perona. Graph-based visual saliency. Advances in Neural Information Processing Systems, 19:545-552, 2007.

[4] L. Y. Zhang, M. H. Tong, et al. . SUN: A bayesian framework for saliency using natural statistics. Journal of Vision, 10(1):1-20, 2008.

[5] X. Hou, L. Zhang. Saliency detection: A spectral residual approach. IEEE Conference on Computer Vision and Pattern Recognition, pages 1-8, 2007.

[6] R. Achanta, S. Hemami, et al. Frequency-tuned salient region detection. IEEE Conference on Computer Vision and Pattern Recognition, 2009.

[7] M. Cheng, G. Zhang, et al.. Global contrast based salient region detection. IEEE Conference on Computer Vision and Pattern Recognition, 2011.

[8] S. Goferman, L. ZELNIK-manor and A.Tal. Context-aware saliency detection. IEEE Conference on Computer Vision and Pattern Recognition, 2010 
[9] V. Gopalakrishnan, Y.Hu and D. Rajan. Random walks on graphs for salient object detection in images. IEEE Trans. on Image Processing, vol.9, no.12, 2012.

[10]C. Yang, L. Zhang, et al.. Saliency detection via graph-based manifold ranking. IEEE Conference on Computer Vision and Pattern Recognition, 2013.

[11] B. Jiang, L. Zhang, et al.. Saliency detection via absorbing markov chain. IEEE Internationa Conference on Computer Vision, 2013.

[12] R. Achanta, K. Smith et al.. Slic superpixel. Technial report, EPFL, Tech.Rep. 149300, 2010.
[13] X. Shen and Y. Wu. A unified approach to salient object detection via low ranking recovery. IEEE Conference on Computer Vision and Pattern Recognition. 2012.

[14] Y. Zhang and M. Shah. Visual attention detection in video sequences using spatiotemporal cues. ACM Multimedia, pages 815-824, 2006.

[15] R. Achanta and S. Süsstrunk. Saliency detection using maximum sysmmetric surround. IEEE, International Conference on Image Processing, 